\title{
Selective control of HOD photodissociation using CW lasers
}

\author{
MANABENDRA SARMA, ${ }^{1}$ S ADHIKARI ${ }^{1,2}$ and MANOJ K MISHRA ${ }^{1, *}$ \\ ${ }^{1}$ Department of Chemistry, Indian Institute of Technology, Powai, Mumbai 400076 \\ ${ }^{2}$ Department of Chemistry, Indian Institute of Technology, Guwahati, North Guwahati 781039 \\ e-mail: mmishra@iitb.ac.in
}

MS received 1 June 2007; accepted 14 July 2007

\begin{abstract}
Selective control of HOD photodissociation ( $\mathrm{H}-\mathrm{O}+\mathrm{D} \leftarrow \mathrm{HOD} \rightarrow \mathrm{H}+\mathrm{O}-\mathrm{D}$ ) has been theoretically investigated using $\mathrm{CW}$ lasers with appropriate carrier frequency and $|0,0\rangle,|0,1\rangle$ and $|0,2\rangle$ with zero quantum of excitation in the $\mathrm{O}-\mathrm{H}$ bond and zero, one and two quanta of excitation in the $\mathrm{O}-\mathrm{D}$ bond as the initial states. Results indicate that the $\mathrm{O}-\mathrm{H}$ bond in HOD can be selectively dissociated with a maximum flux of $87 \%$ in the $\mathrm{H}+\mathrm{O}-\mathrm{D}$ channel from the ground vibrational state $|0,0\rangle$. For the O-D bond dissociation, it requires two quanta of excitation $(|0,2\rangle)$ in the $\mathrm{O}-\mathrm{D}$ mode to obtain $83 \%$ flux in the $\mathrm{H}-\mathrm{O}+\mathrm{D}$ channel. Use of a two colour laser set-up in conjunction with the field optimized initial state (FOIST) scheme to obtain an optimal linear combination of $|0,0\rangle$ and $|0,1\rangle$ vibrational states as the initial state provides an additional $7 \%$ improvement to flux in the $\mathrm{H}-\mathrm{O}+\mathrm{D}$ channel as compared to that from the pure $|0,1\rangle$ state.
\end{abstract}

Keywords. Selective bond-breaking; photodynamic control; photodissociation; CW laser; field optimized initial state (FOIST).

\section{Introduction}

The promise of using ultra short, high intensity lasers as molecular scissors to cleave bonds selectively continues to attract much theoretical and experimental effort. ${ }^{1-6}$ The deuterated water molecule HOD where the $\mathrm{O}-\mathrm{H}$ and $\mathrm{O}-\mathrm{D}$ bonds can be excited separately has been a popular prototype to test a variety of approaches. ${ }^{7-35}$ The first excited state $\tilde{\mathrm{A}}\left({ }^{1} \mathrm{~B}_{1}\right)$ of HOD being totally repulsive, most approaches to selective control of $\mathrm{O}-\mathrm{H}$ and $\mathrm{O}-\mathrm{D}$ dissociation have been based on designing schemes which will transport the molecule to the desired $\mathrm{H}+\mathrm{O}-\mathrm{D} / \mathrm{H}-\mathrm{O}+\mathrm{D}$ region of the repulsive $\tilde{\mathrm{A}}\left({ }^{1} \mathrm{~B}_{1}\right)$ surface using $\mathrm{UV}$ frequencies in the first absorption band. Considerable selectivity in dissociation of the $\mathrm{O}-\mathrm{H}$ bond has been demonstrated even without prior vibrational excitation of the $\mathrm{O}-\mathrm{H}$ bond, ${ }^{8-12,20,30,32-35}$ but in most cases, prior excitation of the $\mathrm{O}-\mathrm{H}$ bond to ensure its deposition in the $\mathrm{H}+\mathrm{O}-\mathrm{D}$ channel on transition to the repulsive $\tilde{A}\left({ }^{1} B_{1}\right)$ surface has been the more favoured route. ${ }^{8-10,12-18,22-24,29}$ In case of selective dissociation of the $\mathrm{O}-\mathrm{D}$ bond via transition to the repulsive first excited surface, prior excitation in the O-D stretch has been necessary. ${ }^{10,12,18,20,21,31,33-35}$

\footnotetext{
*For correspondence
}

Most of the theoretical approaches to selective control of HOD photodissociation have used $\delta$-function or very narrow Gaussian pulses with more or less instant transition of the molecule from ground to the repulsive excited surface. ${ }^{9,10,12,18,29,30}$ This precludes any cross-talk between the two surfaces and the large frequency band width of these narrow pulses does not permit analyses based on manipulation of individual vibrational levels. We have pursued new scenarios $^{33-35}$ which provide selectivity with large yield for dissociation of any of the two $(\mathrm{O}-\mathrm{H} / \mathrm{O}-\mathrm{D})$ bonds using simple Gaussian UV pulses with temporal widths which lead to sufficiently narrow frequency bandwidth to permit mechanistic analysis based on excitation of individual vibrational levels. This approach has revealed new routes to control via manipulation of vibrational states on the ground surface induced by the pulse mediated cross-talk. The frequency band width of our Gaussian pulse is $\sim 1000 \mathrm{~cm}^{-1}$ and since the vibrational separation between levels being manipulated is no less than $2642 \mathrm{~cm}^{-1}\left(V_{02}-V_{01}\right)$ the mechanistic propositions advocated using these pulses are on a relatively sound footing. ${ }^{33,35}$ However, it is clearly useful to examine, if same results can be obtained with $\mathrm{CW}$ lasers with no uncertainty in the frequencies employed. Also, since the temporal width of the Gaussian enve- 
lope requires considerable time lapse before full power of the pulse can come into play it is useful to examine if the faster build up of full power in $\mathrm{CW}$ lasers will also lead to new dynamics allowing desired selectivity with less intense pulses.

Towards this end, it is our purpose in this paper to examine the feasibility of achieving selective dissociation of the desired $\mathrm{O}-\mathrm{H} / \mathrm{O}-\mathrm{D}$ bond using $\mathrm{CW}$ lasers with frequencies and initial states identified as being optimal from earlier pulsed applications. ${ }^{33-35}$ Furthermore, to exploit the dependence of photodissociation outcome on the initial vibrational state subjected to the photolysis pulse, we have been advocating the use of field optimized initial state (FOIST) scheme $^{36-38}$ which attempts to distribute the onus for selective control on both the field attributes and the molecular initial state subjected to the chosen photolysis pulse. The field attributes may be chosen for simplicity and chemical insight, and an optimal initial state is generated using FOIST. The FOIST scheme has been successfully applied for selective control of dissociation products in $\mathrm{HI}$ and $\mathrm{IBr}^{36-38}$ and it is also our purpose in this paper to extend this approach to selective control of HOD photodissociation using $\mathrm{CW}$ lasers and to explore if we can supplant FOIST-based mixing of vibrational states with much easier mixing of laser colours.

The following section presents an outline of computational considerations and is followed by a discussion of results. A summary of main observations concludes this paper.

\section{Method}

The first and second excited electronic states of HOD are well separated and following earlier investigations, we too formulate the HOD dynamics considering only the ground and the first excited electronic state of this molecule. In the first absorption band $\left[\tilde{\mathrm{A}}\left({ }^{1} \mathrm{~B}_{1}\right) \leftarrow \tilde{\mathrm{X}}\left({ }^{1} \mathrm{~A}_{1}\right)\right]$ of $\mathrm{HOD}$, excitation from the ground state $\tilde{X}\left({ }^{1} A_{1}\right)$ to the repulsive first excited state $\widetilde{\mathrm{A}}\left({ }^{1} \mathrm{~B}_{1}\right)$ induces a negligible change in the bending angle. ${ }^{8,9}$ The bending mode is not active in the first absorption band, ${ }^{39}$ and hence the internal kinetic energy operator in terms of the conjugate momenta $\hat{p}_{1}$ and $\hat{p}_{2}$ associated with the $\mathrm{O}-\mathrm{H}\left(r_{1}\right)$ and $\mathrm{O}-\mathrm{D}\left(r_{2}\right)$ stretching co-ordinates respectively, is taken as $^{10,12,13,18,29,30}$

$$
\hat{T}=\frac{\hat{p}_{1}^{2}}{2 \mu_{1}}+\frac{\hat{p}_{2}}{2 \mu_{2}}+\frac{\hat{p}_{1} \hat{p}_{2}}{m_{O}} \cos \theta,
$$

where

$$
\begin{gathered}
\hat{p}_{j}=\frac{\hbar}{i} \frac{\partial}{\partial r_{j}}, j=1,2, \quad \mu_{1}=\frac{m_{H} m_{O}}{m_{H}+m_{O}}, \\
\mu_{2}=\frac{m_{O} m_{D}}{m_{O}+m_{D}},
\end{gathered}
$$

and $\theta$ is the fixed bending angle, $104.52^{\circ}$. The ground $\left(V_{g}\right)^{10,12,40}$ and excited $\left(V_{e}\right)^{41,42}$ Potential Energy Surface (PES), the transition dipole moment surface and the UV-molecule interaction Hamiltonian $H_{u v}(t)$ are same as those employed in other calculations on HOD. ${ }^{12,18}$ The time evolution of the corresponding nuclear motion can then be performed using the time-dependent Schrödinger equation,

$$
i \hbar \frac{\partial}{\partial t}\left(\begin{array}{l}
\Psi_{g} \\
\Psi_{e}
\end{array}\right)=\left(\begin{array}{cc}
\hat{H}_{g} & \hat{H}_{u v}(t) \\
\hat{H}_{u v}(t) & \hat{H}_{e}
\end{array}\right)\left(\begin{array}{l}
\Psi_{g} \\
\Psi_{e}
\end{array}\right),
$$

where $\Psi_{g}=\Psi_{g}\left(r_{1}, r_{2}, t\right)$ and $\Psi_{e}=\Psi_{e}\left(r_{1}, r_{2}, t\right)$ are the wave functions associated with nuclear motion in the ground and first excited state, respectively. $\hat{H}_{g}=\hat{T}+\hat{V}_{g}$ and $\hat{H}_{e}=\hat{T}+\hat{V}_{e}$ are the nuclear Hamiltonians for the two electronic states where $\hat{H}_{u v}(t)$ couples as well as perturbs both the electronic states. We solve (2) with the $(t=0)$ initial condition that the ground state wave function $\Psi_{g}$ is a single, field free, vibrational state or a linear combination of more than one vibrational state(s) of the HOD electronic ground state, and the excited wave function $\Psi_{e}=0$, at $t=0$.

Vibrational eigenfunctions of the ground electronic state of the HOD molecule were obtained using the Fourier grid Hamiltonian (FGH) method ${ }^{43}$ modified for two dimensions. ${ }^{44}$ The vibrational eigenfunctions $(|m, n\rangle)$ of the ground electronic state and the corresponding eigenfrequencies compare quite well with those tabulated in ref. 18.

The propagation of the wavefunctions $\left\{\Psi_{g}(t)\right.$, $\left.\Psi_{e}(t)\right\}$ has been performed using (2) where the effect of kinetic energy operator on the wavefunction is evaluated with a two-dimensional fast Fourier transform $(\mathrm{FFT})^{45}$ and the time propagation is carried out using the Lanczos scheme. ${ }^{46}$ The wave function is represented on a spatial grid spanning $r_{\mathrm{O}-\mathrm{H}} / r_{\mathrm{O}-\mathrm{D}}$ bond lengths between $1 a_{0}$ to $10 a_{0}$ in 128 steps with $\Delta r_{\mathrm{O}-\mathrm{H}}=$ $\Delta r_{\mathrm{O}-\mathrm{D}} \cong 0.0703 a_{0}(\cong 0.0372 \AA)$ and propagation of field effects under the influence of a generic CW laser field $E(t)=A * \cos \omega t$ in time steps of $\Delta t=1$ a.u.

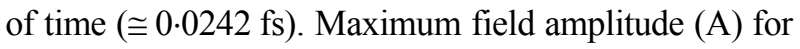


the fields employed in our calculation is $0.05 \mathrm{GV} / \mathrm{cm}$ and maximum field intensity is $2 \cdot 2 \mathrm{TW} / \mathrm{cm}^{2}$. The extent of dissociation of $\mathrm{O}-\mathrm{H}$ and $\mathrm{O}-\mathrm{D}$ modes is determined from flux in the $\mathrm{H}+\mathrm{O}-\mathrm{D}$ and $\mathrm{H}-\mathrm{O}+\mathrm{D}$ channels calculated along asymptotic cuts at $r_{\mathrm{O}-\mathrm{H}}=$ $7 \cdot 5 a_{0}$ and $r_{\mathrm{O}-\mathrm{D}}=7 \cdot 5 a_{0}$ in the $\mathrm{H}+\mathrm{O}-\mathrm{D}$ and $\mathrm{H}-\mathrm{O}+\mathrm{D}$ channels respectively. An absorbing ramp potential is placed immediately thereafter to avoid unphysical reflection from the edges.

In the FOIST scheme, ${ }^{36,38}$ the product yield is maximized through preparation of the initial wavefunction $\left|\Psi_{g},\left(r_{1}, r_{2}\right)\right\rangle$ as a superposition of the field free vibrational wavefunctions $\left\{\psi_{m}\right\}$ of the ground electronic state,

$$
\Psi_{g}(0)=\sum_{m=0}^{M} C_{m} \Psi_{m}
$$

The product yield in the desired channel is related to the time integrated flux $f$ given by,

$$
\begin{aligned}
f= & \int_{0}^{T} d t\langle\Psi(t)|\hat{j}| \Psi(t)\rangle=\int d t\left\langle\Psi_{g}(0)\right| \hat{U}^{\dagger} \\
& (t, 0) \hat{j} \hat{U}(t, 0)\left|\Psi_{g}(0)\right\rangle=\left\langle\Psi_{g}(0)|\hat{F}| \Psi_{g}(0)\right\rangle,
\end{aligned}
$$

with

$$
\Psi(t)=\hat{U}(t, 0) \Psi(0), \quad \hat{F}=\int_{0}^{T} d t \hat{U}^{\dagger}(t, 0) \hat{j} \hat{U}(t, 0),
$$

and

$$
\hat{j}_{i}=\frac{1}{2 \mu_{i}}\left[\hat{p}_{i} \delta\left(r_{i}-r_{i}^{d}\right)+\delta\left(r_{i}-r_{i}^{d}\right) \hat{p}_{i}\right]
$$

where $U$ is the time evolution operator, $\hat{j}_{i}$ is the flux operator in the $i$ th channel and $\mu_{i}, \hat{p}_{i}$ and $r_{i}^{d}$ are the reduced mass, the momentum operator and a grid point in the asymptotic region of the $i$ th channel denoted by reaction coordinate $r_{i}$, with $\mathrm{H}+\mathrm{O}-\mathrm{D}$ channel labelled as 1 and $\mathrm{H}-\mathrm{O}+\mathrm{D}$ channel as 2. The expressions for the total flux $J$ in the $\mathrm{H}+\mathrm{O}-\mathrm{D}$ and $\mathrm{H}-\mathrm{O}+\mathrm{D}$ channels are given by,

$$
\begin{aligned}
J_{H+O-D}= & \int_{0}^{r_{2 d}} \int_{0}^{T} \Psi *\left(r_{1}, r_{2}, t\right)\left(\hat{j}_{1}+\frac{\mu_{2} \cos \theta}{m_{o}} \hat{j}_{2}\right) \\
& \Psi\left(r_{1}, r_{2}, t\right) d r_{2} d t,
\end{aligned}
$$

$$
\begin{gathered}
J_{\mathrm{H}-\mathrm{O}+\mathrm{D}}=\int_{0}^{r_{1}} \int_{0}^{T} \Psi *\left(r_{1}, r_{2}, t\right)\left(\hat{j}_{2}+\frac{\mu_{1} \cos \theta}{m_{0}} \hat{j}_{1}\right) \\
\Psi\left(r_{1}, r_{2}, t\right) d r_{1} d t
\end{gathered}
$$

where the second operator in (5) and (6) represents the effect of kinetic coupling between the $\mathrm{O}-\mathrm{H}$ and O-D modes. The field dependence of $H\left(\vec{r}_{1}, \vec{r}_{2}, t\right)$ manifests itself through $U(t, 0) \cong e^{-i H t / \hbar}$ where $H=H_{\text {molecule }}+H_{\mathrm{UV}}$. Optimization of the channel and field specific flux functional $\langle\Psi(0)|\hat{F}| \Psi(0)\rangle$ with respect to the coefficients $C_{m}$ employed in (3) leads to the Rayleigh-Ritz eigenvalue problem ${ }^{36,37}$

$$
\mathbf{F C}=\mathbf{f C}
$$

where the $\mathbf{f}$ is the diagonal matrix of the time integrated flux matrix $\mathbf{F}$. The matrix elements of $\mathbf{F}$ in the $i$ th channel are given by ${ }^{38}$

$$
F_{k l}^{i} \approx \Delta t \sum_{n=0}^{N_{t}}\left\langle\psi_{k}(n \Delta t)\left|J_{i}\right| \psi_{l}(n \Delta t)\right\rangle
$$

where

$$
J_{1}=\left(\hat{j}_{1}+\frac{\mu_{2} \cos \theta}{m_{0}} \hat{j}_{2}\right) \text { and } J_{2}=\left(\hat{j}_{2}+\frac{\mu_{1} \cos \theta}{m_{0}} \hat{j}_{1}\right) .
$$

We propagate the $M$ initial states, included in the expansion manifold of (3) using spatial and temporal details presented earlier and calculate accumulated flux matrices $\left(F_{k l}^{i}\right)$ both for the $\mathrm{H}+\mathrm{O}-\mathrm{D}$ and $\mathrm{H}-\mathrm{O}+\mathrm{D}$ dissociation channels. The accumulated $F_{k l}^{(\mathrm{H}+\mathrm{O}-\mathrm{D})}$ or $F_{k l}^{(\mathrm{H}-\mathrm{O}+\mathrm{D})}$ matrices are diagonalized and eigenvector $\left(C_{m}^{\max }\right)$ corresponding to the highest eigenvalue $f_{\max }$ indicates the maximum possible dissociation yield available for the field and the manifold of vibrational eigenstates chosen in that calculation. The $C_{m}^{\max }$ defines the initial wavefunction, $\Psi_{g}(0)=\sum_{m} C_{m}^{\max } \Psi_{m}$ which will provide $\left(f_{\max } \times 100 \%\right)$ dissociation in the chosen channel $\left(\mathrm{H}+\mathrm{O}-\mathrm{D}\right.$ for $J_{1}$ or $\mathrm{H}-\mathrm{O}+\mathrm{D}$ for $\left.J_{2}\right)$ for the field used in $H_{u v}(t)$ and the expansion manifold of field free vibrational states $(M)$ utilized in (3).

Selectivity and yield can also be influenced by supplementing or substituting the mixing of vibrational states by mixing additional colours (frequencies) $)^{38}$ to the resonant carrier frequency with a single or multicolour field $E(t)=A^{*} \sum_{i} \cos \omega_{i} t$ where $\omega_{i}$ are physically motivated UV frequencies that induce transitions to same final state from different vibra- 
tional levels of the ground state. The frequencies in this multicolour field are well separated so that mechanistic insights in terms of excitation from and dumping to specific vibrational levels may be attempted. Initial results from first application of these ideas to the selective control of bond dissociation in HOD are presented in the following section.

\section{Results and discussion}

As mentioned earlier, it is our purpose in this paper to tap into the monochromaticity and quicker access to full power provided by $\mathrm{CW}$ lasers to examine the changes in dynamical details and feasibility of achieving selectivity and yield in the dissociation of $\mathrm{O}-\mathrm{H}$ and $\mathrm{O}-\mathrm{D}$ bonds of HOD using frequencies which have provided best results from our earlier pulsed laser applications. ${ }^{33-35}$ Towards this end, we present results from subjecting the HOD molecule to $\mathrm{CW}$ laser $E(t)=0 \cdot 1 * \cos \omega t$ where $\omega=71361 \mathrm{~cm}^{-1}$ in figure 1. As expected, with quicker access to full power, the population transfer between the ground and excited states is indeed fast and there is continuous build up of flux in the $\mathrm{H}+\mathrm{O}-\mathrm{D}$ and $\mathrm{H}-\mathrm{O}+\mathrm{D}$ channels with a time lag of mere $15 \mathrm{fs}$. Even with the low intensity of $2.2 \mathrm{TW} / \mathrm{cm}^{2}$ there is more or less complete dissociation with $\sim 87.2 \%$ flux in $\mathrm{H}+\mathrm{O}-\mathrm{D}$ channel and $\sim 11.3 \%$ in the $\mathrm{H}-\mathrm{O}+\mathrm{D}$ channel which provides an additional $5 \%$ flux in the

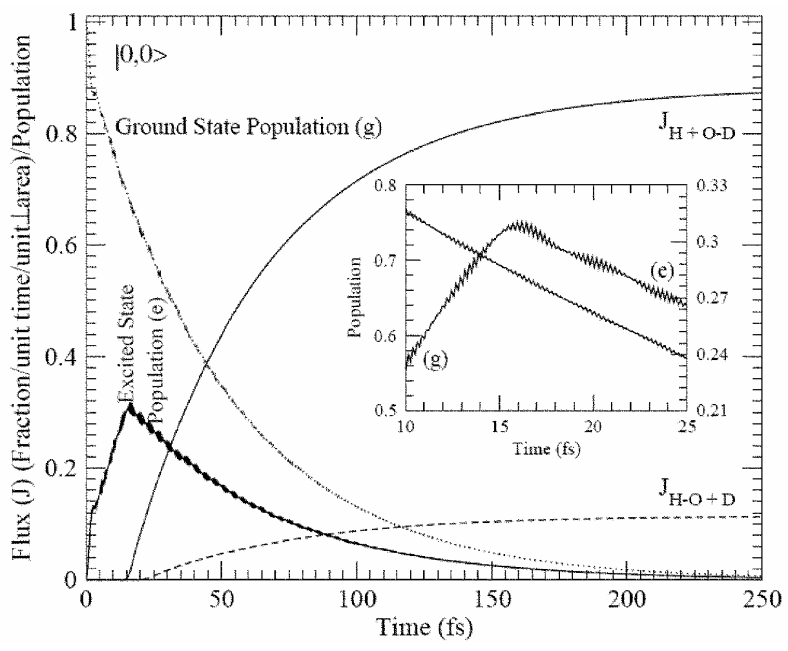

Figure 1. Ground and excited state populations and $\mathrm{H}+\mathrm{O}-\mathrm{D}$ and $\mathrm{H}-\mathrm{O}+\mathrm{D}$ flux using $|0,0\rangle$ as initial state with CW laser field $E(t)=0.01 * \cos \omega t$ where $\omega=71361 \mathrm{~cm}^{-1}$. Details of population transfer between ground and excited electronic states are presented in the inset.
$\mathrm{H}+\mathrm{O}-\mathrm{D}$ channel compared to that obtained with the 50 fs pulsed laser. ${ }^{33-35}$ As can be seen from the inset, there is a cross-talk between the two surfaces, even for the low intensity CW laser field employed
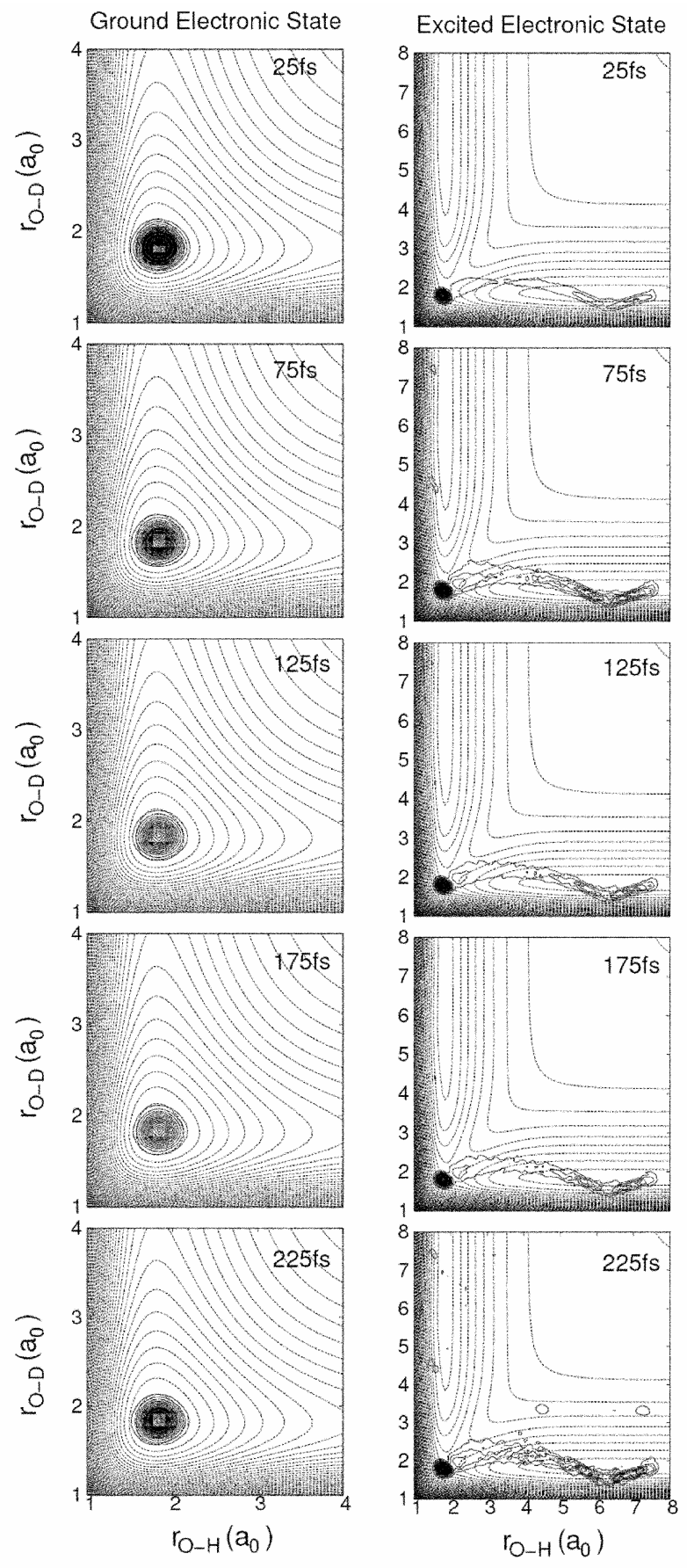

Figure 2. Time evolution of $|0,0\rangle$ on ground and excited electronic state with the CW laser field utilized in figure 1. 
here. The assumption of field induced instantaneous transport of the molecule to the excited surface with no role for cross-talk between the two surfaces in the dynamical outcome ${ }^{8-10,12,13,16,23,26,30-32}$ is not borne out by results of figure 1 . In figure 2 , where we have mapped the population dynamics and flow in detail. As can be seen from figure 2, there is a change in the spatial profile of the probability density on the ground state and this spatial steering of the ground state probability density we feel, contributes to the preferred transport to the $\mathrm{H}+\mathrm{O}-\mathrm{D}$ region of the upper surface, specially since there is considerable bouncing off the upper portions of the walls in the $\mathrm{H}+\mathrm{O}-\mathrm{D}$ channel at longer times.

For selective dissociation of the $\mathrm{O}-\mathrm{D}$ bond, prior excitation in this mode either through prior preparation $^{10,12,13,18,20,21,23,31}$ or field-induced manipulations using two colour lasers ${ }^{33,35}$ were required both theoretically ${ }^{10,12,13,18,20,23,31}$ and experimentally. ${ }^{21,31}$ As can be seen from figures 3 and 4 , subjecting the $|0,2\rangle$ state with 2 quanta of excitation in the $\mathrm{O}-\mathrm{D}$ bond to the CW laser $E(t)=0 \cdot 1 * \cos \omega t$ with $\omega=54192 \mathrm{~cm}^{-1}$ does produce much more flux in the $\mathrm{H}-\mathrm{O}+\mathrm{D}$ channel $(\sim 82.6 \%)$, and the prior stretching of the $\mathrm{O}-\mathrm{D}$ bond (figure 4) helps concentrate the probability density flow in the $\mathrm{H}-\mathrm{O}+\mathrm{D}$ channel on the repulsive excited surface. The flux in the $\mathrm{H}-\mathrm{O}+\mathrm{D}$ channel picks up almost simultaneously with that in the kinematically favoured $\mathrm{H}+\mathrm{O}-\mathrm{D}$ channel and surges ahead

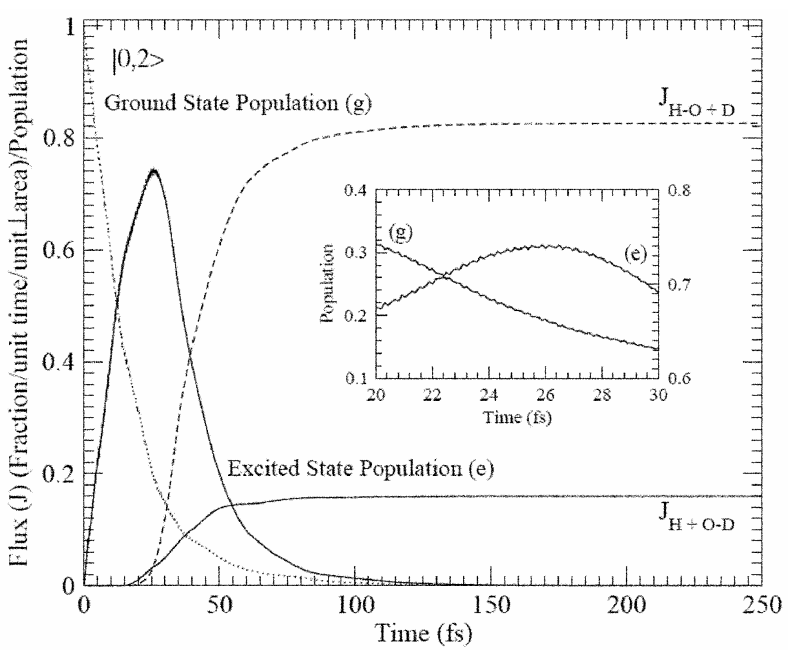

Figure 3. Ground and excited state populations and $\mathrm{H}+\mathrm{O}-\mathrm{D}$ and $\mathrm{H}-\mathrm{O}+\mathrm{D}$ flux using $|0,2\rangle$ as initial state with the same field profile mentioned in figure 1 except the carrier frequency $\omega=54192 \mathrm{~cm}^{-1}$. Details of population transfer between ground and excited electronic states are presented in the inset. despite some synchronous cross-talk between the ground and excited surfaces (figure 3 and inset). Once again, the selectivity with very large yield achieved using a low intensity $\mathrm{CW}$ laser compares
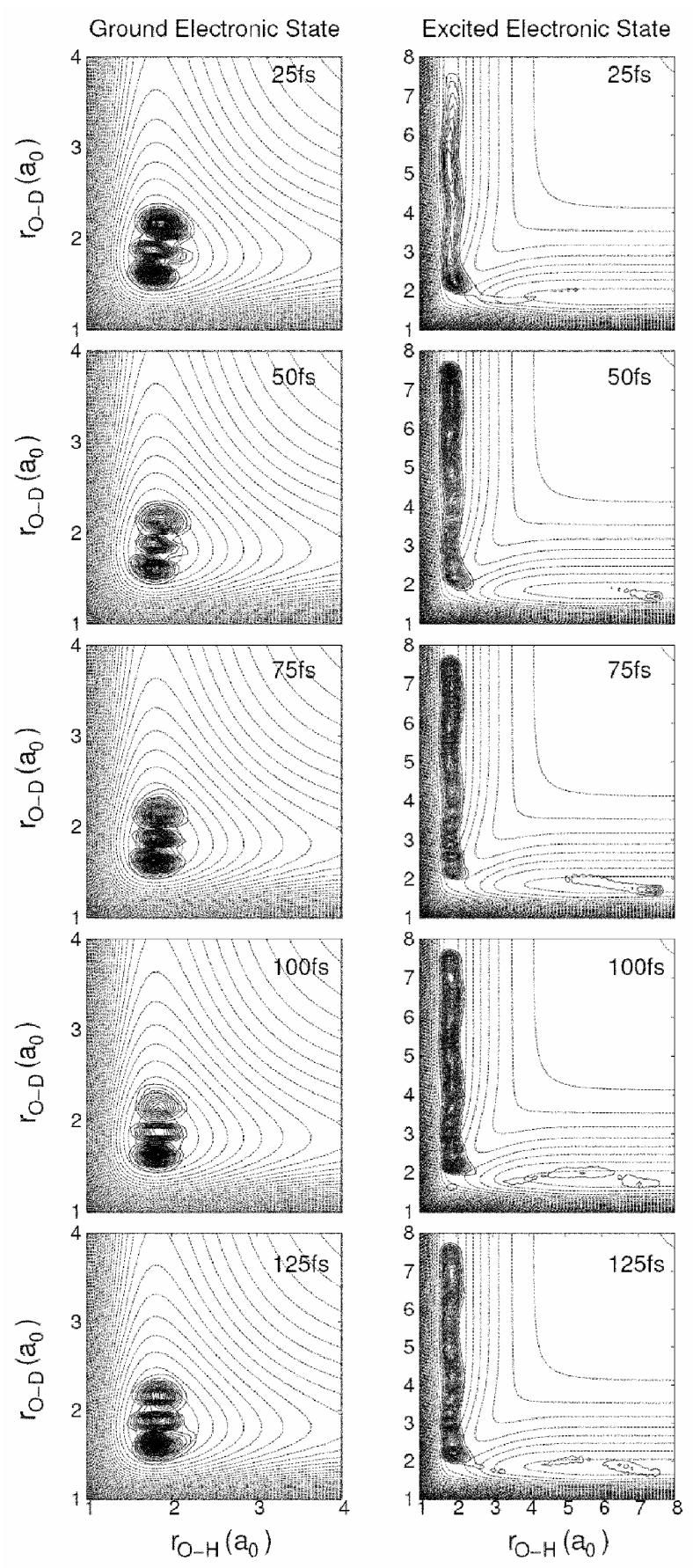

Figure 4. Time evolution of $|0,2\rangle$ on ground and excited electronic state with the field profile mentioned in figure 3 . 
Table 1. Flux obtained using different initial states and $\mathrm{CW}$ laser frequencies.

\begin{tabular}{lccc}
\hline Initial state(s) & Frequency $\left(\mathrm{cm}^{-1}\right)$ & $\mathrm{H}+\mathrm{O}-\mathrm{D}$ Flux $(\%)$ & $\mathrm{H}-\mathrm{O}+$ D Flux (\%) \\
\hline$|0,0\rangle$ & 71361 & $87 \cdot 18$ & $11 \cdot 26$ \\
$|0,1\rangle$ & 59703 & $39 \cdot 26$ & $58 \cdot 97$ \\
$|0,2\rangle$ & 54192 & $16 \cdot 00$ & $82 \cdot 56$ \\
$C_{1}|0,0\rangle+C_{2}|0,1\rangle$ & 59703 & $38 \cdot 84$ & $59 \cdot 39$ \\
$C_{1}=(-0 \cdot 101988,0 \cdot 056327), C_{2}=(0 \cdot 993189,0 \cdot 000000)$ & & $66 \cdot 26$ \\
$C_{1}|0,0\rangle+C_{2}|0,1\rangle$ & 60277 and 59703 & $31 \cdot 72$ & \\
$C_{1}=(-0 \cdot 193577,0 \cdot 075161), C_{2}=(0 \cdot 978282,0 \cdot 000000)$ & & \\
\hline
\end{tabular}

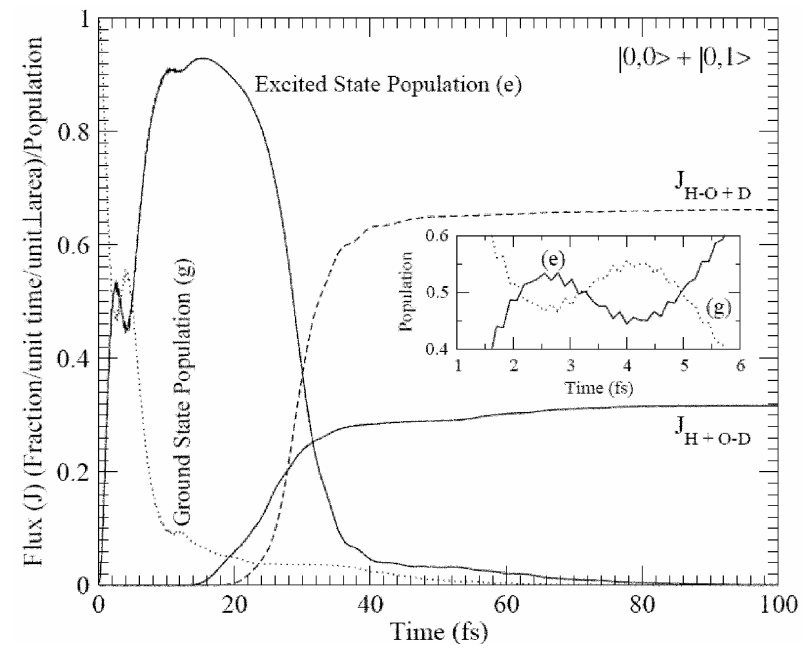

Figure 5. Ground and excited state populations and $\mathrm{H}+\mathrm{O}-\mathrm{D}$ and $\mathrm{H}-\mathrm{O}+\mathrm{D}$ flux using the FOIST optimized $|0,0\rangle+|0,1\rangle$ combination (with mixing coefficients listed in table 1) as initial state with $\mathrm{CW}$ laser field $E(t)=0.01 *\left(\cos \omega_{1} t+\cos \omega_{2} t\right)$ with $\omega_{1}=60277 \mathrm{~cm}^{-1}$ and $\omega_{2}=59703 \mathrm{~cm}^{-1}$. Inset has the more resolved structure of population transfer between ground and excited electronic states.

favourably with those from higher intensity pulsed laser. $^{33-35}$

A more complicated dynamical pattern is witnessed in population transfer and flux plots of figure 5 and probability density plots of figure 6 from exposure of a FOIST optimized $|0,1\rangle+|0,1\rangle$ combination exposed to a two colour $\mathrm{CW}$ laser set-up $E(t)=$ $0.01 *\left(\cos \omega_{1} \mathrm{t}+\cos \omega_{2} t\right)$ with $\omega_{1}=60277 \mathrm{~cm}^{-1}$ and $\omega_{2}=59703 \mathrm{~cm}^{-1}$. This combination of $|0,0\rangle$ and $|0,1\rangle$ states is chosen to mitigate the difficulty associated with preparation of a pure $|0,2\rangle$ state whose dynamics was charted in figures 3 and 4 and the two colours $\left(\omega_{1}\right.$ and $\left.\omega_{2}\right)$ have been chosen as $\omega_{1}$ is close to the frequency $\left(60777 \mathrm{~cm}^{-1}\right)$ which maximizes $\mathrm{H}-\mathrm{O}+\mathrm{D}$ flux from the $|0,0\rangle$ state and the second colour, $\omega_{2}$, provides maximum flux in the $\mathrm{H}-\mathrm{O}+\mathrm{D}$ channel with $|0,1\rangle$ as the initial state (table 1 ). The $\omega_{1}$ and $\omega_{2}$ combination provides the highest
$\mathrm{H}-\mathrm{O}+\mathrm{D}$ flux obtained using a two colour CW laser set-up and a FOIST optimized $|0,0\rangle+|0,1\rangle$ initial state. The ensuing results show a pronounced exchange of population between the ground and excited states with a synchronized cross-talk between the two levels (figure 5 inset) in early stages providing for a complicated mix of probability density in the 10 fs probability density plot on the ground surface in figure 6 . The initial bias in favour of $\mathrm{H}-\mathrm{O}+\mathrm{D}$ channel introduced by the $|0,1\rangle$ component leads to larger flux in the $\mathrm{H}-\mathrm{O}+\mathrm{D}$ channel (figure 6) as desired but as can be seen, there is considerable modification of nodal topology in the $\mathrm{O}-\mathrm{H}$ mode as well and the flux in $\mathrm{H}+\mathrm{O}-\mathrm{D}$ channel is as large as $\sim 31 \cdot 7 \%$. A summary of these results is collected in table 1 .

It is also worth mentioning that the two colours in our FOIST-based selective control scheme have no phase difference between them. The phase difference between the two colours can be another important tool for selective cleavage of bonds. The importance of this has already been seen in the extensive work of Brumer and Shapiro ${ }^{3}$ and we have therefore not explored this issue. The phase difference between the two colours could add to both selectivity and yield and may be gainfully employed in a future study.

\section{Conclusions}

A 2-surface fully quantum dynamical treatment of $\mathrm{H}-\mathrm{O}-\mathrm{D}$ molecule's interaction with different initial states and carrier frequencies provides new routes for selective control and insights into micro dynamic-details behind a small number of control pathways explored here. The results presented here show that low intensity $\mathrm{CW}$ lasers can be as effective as the higher intensity pulsed lasers in some cases but initial results from a 2 colour $\mathrm{CW}$ laser based FOIST approach needs more extensive mapping of frequency dependence to achieve results obtained with pulsed laser. ${ }^{35}$ 


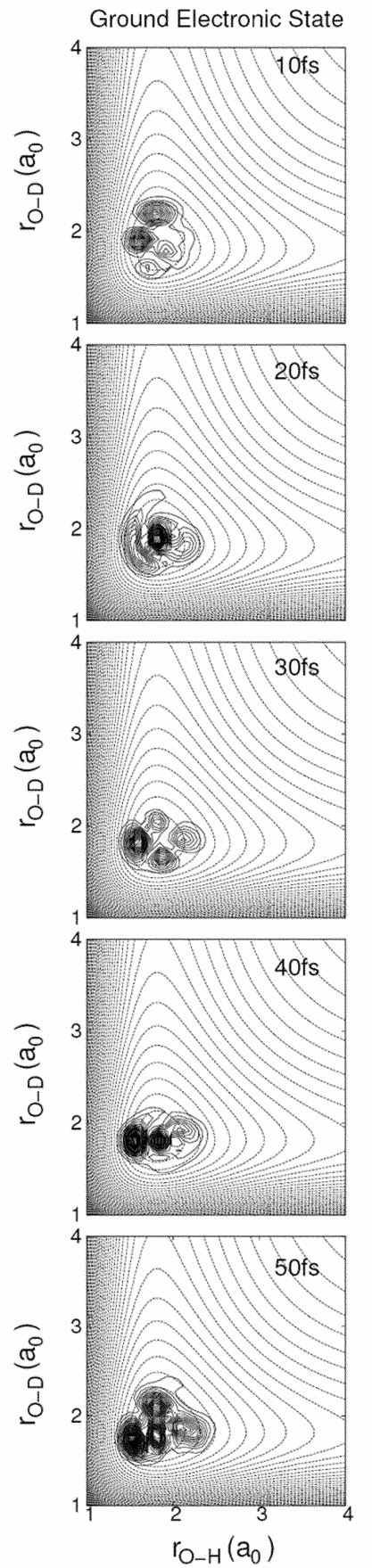

Excited Electronic State
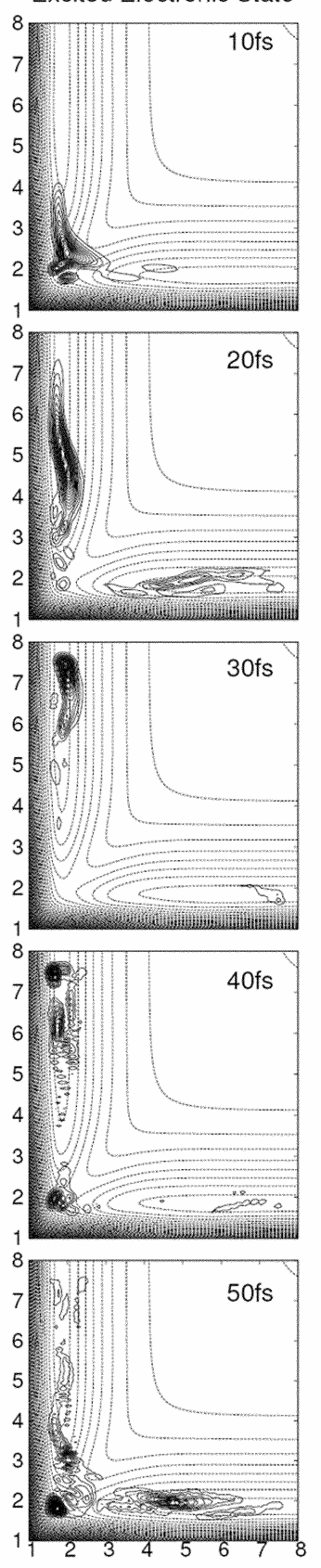

$\mathrm{r}_{\mathrm{O}-\mathrm{H}}\left(\mathrm{a}_{0}\right)$

Figure 6. Time evolution of the optimal (FOIST) $|0,0\rangle+|0,1\rangle$ combination on ground and excited electronic states with the field profile mentioned in figure 5 .

A feature that has not been analysed is the robustness of FOIST-based optimal linear combination of states. This issue has been dealt with elsewhere ${ }^{47}$ and a more detailed investigation and analysis of other routes to selective bond cleavage is underway in our group.

\section{Acknowledgement}

M K M acknowledges financial support from the Department of Science and Technology (DST) and Board of Research in Nuclear Sciences (BRNS) of the Department of Atomic Energy (DAE), India. Manabendra Sarma acknowledges support from Council of Scientific and Industrial Research (CSIR). S A acknowledges DST, Government of India for partial financial support.

\section{References}

1. Rabitz H 2006 Science 314264

2. Elles C G and Crim F F 2006 Annu. Rev. Phys. Chem. 57273

3. Shapiro M and Brumer P 2003 Principles of the quantum control of molecular processes (New York: Wiley)

4. Henriksen N E 2002 Chem. Soc. Rev. 3137

5. Rice S A 2001 Nature (London) 409422

6. Rice S A and Zhao M 2000 Optical control of molecular dynamics (New York: Wiley)

7. Segev E and Shapiro M 1982 J. Chem. Phys. 775604

8. Engel V and Schinke R 1988 J. Chem. Phys. 886831

9. Zhang J and Imre D G 1988 Chem. Phys. Lett. 149 233

10. Zhang J, Imre D G and Frederick J H 1989 J. Phys. Chem. 931840

11. Shafer N, Satyapal S and Bershon R 1989 J. Chem. Phys. 906807

12. Imre D G and Zhang J 1989 Chem. Phys. 13989

13. Hartke B, Manz J and Mathis J 1989 Chem. Phys. 139123

14. Vander Wal R L, Scott J L and Crim F F $1990 \mathrm{~J}$. Chem. Phys. 92803

15. Bar I, Cohen Y, David D, Rosenwaks S and Valentini J J 1990 J. Chem. Phys. 932146

16. Vander Wal R L, Scott J L, Crim F F, Weide K and Schinke R 1991 J. Chem. Phys. 943548

17. Bar I, Cohen Y, David D, Arusi-Parper T, Rosenwaks S and Valentini J J 1991 J. Chem. Phys. 95 3341

18. Amstrup B and Henriksen N E 1992 J. Chem. Phys. 978285

19. Shapiro M and Brumer P 1993 J. Chem. Phys. 98201

20. Henriksen N E and Amstrup N E 1993 Chem. Phys. Lett. 21365

21. Cohen Y, Bar I and Rosenwaks S 1995 J. Chem. Phys. 1023612

22. Brouard M and Langford S R 1997 J. Chem. Phys. 1066354

23. Campolieti G and Brumer P 1997 J. Chem. Phys. 107 791 
24. Meyer S and Engel V 1997 J. Phys. Chem. A101 7749

25. Plusquellic D F, Votava O and Nesbitt D J $1998 \mathrm{~J}$. Chem. Phys. 1096631

26. Schröder T, Schinke R, Ehara M and Yamashita K 1998 J. Chem. Phys. 1096641

27. Mishima K and Yamashita K 1999 J. Chem. Phys. 1107756

28. Yang H F, Hwang D W, Lin J J and Ying X $2000 \mathrm{~J}$. Chem. Phys. 11310597

29. Elghobashi N, Krause P, Manz J and Oppel M 2003 Phys. Chem. Chem. Phys. 54806

30. Henriksen N E, Möller K B and Engel V $2005 \mathrm{~J}$. Chem. Phys. 122204320

31. Akagi H, Fukazawa H, Yokoyama K and Yokoyama A 2005 J. Chem. Phys. 123184305

32. Möller K B, Westtoft H C and Henriksen N E 2006 Chem. Phys. Lett. 41965

33. Sarma M, Adhikari S and Mishra M K 2006 Chem. Phys. Lett. 420321

34. Adhikari S, Deshpande S, Sarma M, Kurkal V and Mishra M K 2006 Radiat. Phys. Chem. 752106

35. Sarma M, Adhikari S and Mishra M K 2007 J. Chem. Phys. 127024305
36. Vandana K and Mishra M K 1999 J. Chem. Phys. 110 5140

37. Vandana K and Mishra M K 1999 Adv. Quant. Chem. 35261

38. Vandana K and Mishra M K 2000 J. Chem. Phys. 113 2336

39. Sension R J, Brudzynski R J and Hudson B 1988 Phys. Rev. Lett. 61694

40. Reimers J R and Watts R O 1984 Mol. Phys. 52357

41. Staemmler V and Palma A 1985 Chem. Phys. 9363

42. Engel V, Schinke R and Staemmler V 1988 J. Chem. Phys. 88129

43. Marston C C and Balint-Kurti G G 1989 J. Chem. Phys. 913571

44. Dutta P, Adhikari S and Bhattacharyya S P 1993 Chem. Phys. Lett. 212677

45. Kosloff D and Kosloff R 1983 J. Comput. Phys. 52 35

46. Leforestier C, Bisseling R H, Cerjan C, Feit M D, Friesner R, Guldberg A, Hammerich A, Jolicard G, Karralein W, Meyer H D, Lipkin M, Roncero O and Kosloff R 1991 J. Comput. Phys. 9459

47. Sarma M and Mishra M K (to be submitted) 